\title{
Existence \& Uniqueness of Consistent Conjectural Variation Equilibrium in Electricity Markets
}

\author{
Youfei Liu, Y.X.Ni, Senior Member, IEEE
}

\begin{abstract}
The real electricity markets are usually oligopoly, where market suppliers (generators) will have some market power and can adopt strategic bidding strategies for maximum profits. Generally, the game-theory based methods are the natural way to analyze the market equilibrium and study the strategic behaviors. As a widely studied method in game theory, the conjecture variation technique is reported to model the strategic behavior in deregulated electricity markets recently. Unfortunately, the conjecture variation models have been criticized for the drawback of logical inconsistence and abundant equilibria. Aim for this, the existence and uniqueness of consistent conjectural variation equilibrium in the electricity markets are investigated. Due to some good characteristics of electricity markets and using an infinite horizon optimization model, it is shown that the consistent conjecture variation will satisfy a coupled nonlinear equation system and there is only one equilibrium.
\end{abstract}

Index terms - Electricity markets, Oligopoly, Consistent conjecture variation, Existence \& uniqueness

\section{INTRODUCTION}

D UO to the well-known reasons of various market barriers, such as the long construction period and huge capital investment, the real electricity markets are more akin to the oligopoly, which means that individual market supplier (generator) will have some market power and can manipulate the market price in some extent through his behavior. Such a fact brings up the problem of strategic bidding ${ }^{[1]}$. Generally, the bidding in electricity markets can be modeled as a supplier game, and gametheory based methods are applied to analyze the market equilibrium and study the strategic behaviors ${ }^{[2][3][4][5]}$, where the essence of strategic bidding is the study on how to exploit and utilize the market power either explicitly or implicitly.

Recently, the conjectural variation (CV)-based method, a well studied model in game theory, is also applied for the study of generators' strategic behavior in electricity markets ${ }^{[6[7][8][9]}$. The conjectural variation is defined as one's belief or expectation on the rival's reaction to his decision ${ }^{[10]}$. Hence, it can be expected that, after taking into account of the conjecture about the rivals' response, the $\mathrm{CV}$ model will provide a better way to study the suppliers' strategic behaviors. First in the literature of electricity engineering, [6] applied the conjectural variation approach to simulate the Spain

Youfei Liu is with the EEE Department, Hong Kong University, Hong Kong.(e-mail: youfei@eee.hku.hk)

Y.X.Ni is with the EEE Department, Hong Kong University, Hong Kong.(e-mail:yxni@eee.hku.hk) electricity markets, and then [7] applied it to simulate the England \& Wales electricity markets. [8] studied the relationship between the conjectural variation approach and the classical game-theory bidding strategies. Furthermore, for the situation of repeated hourly bidding in electricity markets, [9] tried to introduce the dynamic conjecture variation approach. All of them have presented us the successful applications of conjecture variation approach.

However, the world is never perfect. For the conjecture variation approach, some fundamental problems still remain unsolved. The major arguments against the $\mathrm{CV}$ method are: (1) the conjecture variation may be logically inconsistent because supplier may have expectations about how his rivals will behave that need not to be correct, or suppliers are 'right for wrong reason'; (2) there may be abundant of equilibrium. The criticism on inconsistent conjecture variation spurred the modern searches for "Consistent conjectures" [11][12][13][14] Generally, a conjectural variation is consistent if it is equivalent to the actual response taken by his rivals. But the abundance of equilibrium still makes trouble. The multiplicity of equilibrium raises a question about the very meaning of equilibrium and which equilibrium should be reached. Although, the requirement of consistent conjectures will limit this unwanted abundance of equilibrium, there is still a strong need to solve the uniqueness of equilibrium, because the unique equilibrium can solve the problem of many oligopoly solution concepts by determining a single equilibrium price and quantity. To author's knowledge, there is still no rigorous mathematical proof on the uniqueness of consistent conjecture variation equilibrium in a general oligopoly market. For some unknown reason, most of done work just skips this issue ${ }^{[8][9][14]}$. Fortunately, given some good characteristics of electricity markets, such as increasing quadratic cost function and down-slope linear demand function, it is possible to obtain some nice result.

Given this background, it is the aim of this paper to focus on the existence and uniqueness of consistent conjecture variation equilibrium in the oligopolistic electricity markets. It is assumed here that each supplier has the perfect information about how his rivals will response to his action. And particularly in our formulation, the conjecture variation is taken as the supplier's decision variable, and a dynamic adjustment process is proposed to maximize the profits. Based on Pontryagin's maximum principle, the consistent conjecture variations are formulated as a coupled nonlinear equation system. Then through the fixed-point theory, it is rigorously proved that there is only unique conjecture variation equilibrium, and 
in equilibrium, it results a more competitive behavior than the Cournot competition.

This paper is organized as follows. With the consideration of increasing quadratic cost functions and down-slope linear demand function in electricity markets, Section II gives the basic formulation of consistent conjecture variation equilibrium. Then in section III, it is proved that that there is only unique conjecture variation equilibrium. Section VI gives the numerical example with conclusions in section $\mathrm{V}$.

\section{MODELS}

Considering an oligopolistic electricity market with $n$ suppliers (generators), and the system demand is modeled by an inverse demand function which takes the form:

$$
p=e-f D=e-f \sum_{i=1}^{n} q_{i}
$$

Where $D$ : total demand; $e, f$ : positive coefficients, publicly known for every supplier; $q_{i}$ : generation of supplier $i ;$ for the market balance condition $Q=\sum_{i=1}^{n} q_{i}=D$.

Each supplier has the quadratic production cost function which takes the form:

$$
C_{i}\left(q_{i}\right)=a_{i}+b_{i} q_{i}+\frac{1}{2} c_{i} q_{i}^{2}, \quad i=1, \cdots, n
$$

Where $a_{i}, b_{i}, c_{i}$ : parameters of the cost function, positive.

With the assumption of non-cooperative competition, the individual supplier's profit is the revenue minus the cost:

$$
\pi_{i}=p q_{i}-C_{i}\left(q_{i}\right)
$$

Each supplier (generator) is a profit-maximizer. In order to rationally maximize his profit, each supplier should take into account of the rivals' response to his action when making the generation decision. Such a belief or expectation on the rivals' reaction to ones' own generation decision is defined as 'conjectural variation'. For example, given supplier $i$, his conjecture on supplier $j$ 's variation as a reaction to his output change is defined as ${ }^{[10]}$ :

$$
C V_{i j}=\frac{\partial q_{j}}{\partial q_{i}}
$$

A useful concept is the integrated response of supplier $i$ 's rivals:

$$
C V_{i}=\sum_{j=1, j \neq i}^{n} C V_{i j}=\sum_{j=1, j \neq i}^{n} \frac{\partial q_{j}}{\partial q_{i}}
$$

With (3) and (5), yield:

$$
\begin{aligned}
& \frac{\partial \pi_{i}}{\partial q_{i}}=e-f \sum_{j=1}^{n} q_{j}+\left(-f-f \sum_{j \neq i}^{n} \frac{\partial q_{j}}{\partial q_{i}}\right) q_{i}-\left(b_{i}+c_{i} q_{i}\right) \\
& =e-b_{i}-\left(2 f+c_{i}\right) q_{i}-f \sum_{j \neq i, j=1}^{n} q_{j}-f C V_{i} q_{i}
\end{aligned}
$$

Then based on the first-order condition (6) for optimality, a dynamic adjustment process for each supplier is proposed:

$$
\begin{aligned}
& \dot{q}_{i}=\beta_{i} \frac{d \pi_{i}}{d q_{i}} \\
& =\beta_{i}\left(e-b_{i}-\left(2 f+c_{i}\right) q_{i}-f \sum_{j \neq i, j=1}^{n} q_{j}-f C V_{i} q_{i}\right)
\end{aligned}
$$

Where $\beta_{i}$ is a positive parameter, representing the speed of adjustment. Equation (7) also means that supplier makes the generation decision in the direction of increasing profits.

Actually, the conjecture variation $C V_{i}$ can be handled as a decision variable. Doing so, define a control variable $u_{i}=f C V_{i} q_{i}$, and assume that supplier's objective is to maximize the discounted stream of profits over an infinite planning period, i.e.:

$$
\begin{aligned}
& \max \prod_{i}=\int_{0}^{\infty} e^{-r t}\left[p q_{i}-C_{i}\left(q_{i}\right)\right] d t \\
& =\int_{0}^{\infty} e^{-r t}\left[\left(e-f\left(q_{i}+\sum_{j \neq i}^{n} q_{j}\right)\right) q_{i}-\left(a_{i}+b_{i} q_{i}+\frac{1}{2} c_{i} q_{i}^{2}\right)\right] d t
\end{aligned}
$$

Subject to the state equation:

$$
\begin{aligned}
& \dot{q}_{i}=\beta_{i}\left(e-b_{i}-\left(2 f+c_{i}\right) q_{i}-f \sum_{j \neq i, j=1}^{n} q_{j}-u_{i}\right) \\
& \dot{q}_{j}=\beta_{j}\left(e-b_{j}-\left(2 f+c_{j}\right) q_{j}-f \sum_{k \neq j, k=1}^{n} q_{k}-u_{k}\right) \\
& j=1, \cdots, n, j \neq i
\end{aligned}
$$

Where $r$ is the common discount rate.

For the above equation system, the Pontryagin's maximum principle gives the necessary conditions for optimality. The current-value-Hamiltonian for supplier $i$ is given as:

$$
\begin{aligned}
& H_{i}=\left[\left(e-f\left(q_{i}+q_{-i}\right)\right) q_{i}-\left(a_{i}+b_{i} q_{i}+\frac{1}{2} c_{i} q_{i}^{2}\right)\right] \\
& +\lambda_{i}(t)\left[-\left(2 f+c_{i}\right) q_{i}-f \sum_{j \neq i}^{n} q_{j}(t)-u_{i}(t)+e-b_{i}\right] \\
& +\sum_{j \neq i}^{n} \lambda_{j}(t)\left[-\left(2 f+c_{j}\right) q_{j}-f q_{i}-f \sum_{k \neq j, j \neq i}^{n} q_{k}(t)-u_{j}(t)+e-b_{j}\right]
\end{aligned}
$$

Then the necessary conditions for optimality are:

$$
\begin{gathered}
\dot{q}_{i}=\frac{\partial H_{i}}{\partial \lambda_{i}}, \dot{q}_{j}=\frac{\partial H_{j}}{\partial \lambda_{j}},(j=1, \cdots, n, j \neq i) \\
\frac{\partial H_{i}}{\partial u_{i}}=0 \Rightarrow \lambda_{i}=0 \\
\dot{\lambda}_{i}=r \lambda_{i}-\frac{\partial H_{i}}{\partial q_{i}}=r \lambda_{i}+\left(2 f+c_{i}\right) q_{i}+f \sum_{j \neq i}^{n} q_{j}+b_{i}+f \sum_{j \neq i}^{n} \lambda_{j} \\
\dot{\lambda}_{j}=r \lambda_{j}-\frac{\partial H_{i}}{\partial q_{j}}
\end{gathered}
$$

Where 
$\frac{\partial H_{i}}{\partial q_{i}}=-\left(2 f+c_{i}\right) q_{i}-f \sum_{j \neq i}^{n} q_{j}+e-b_{i}+\lambda_{\iota}\left(-\left(2 f+c_{i}\right)-\frac{\partial u_{i}}{\partial q_{i}}\right)-f \sum_{j \neq i}^{n} \lambda_{j}$.

With equation (12) and (13), we can obtain:

$$
\frac{\partial H_{i}}{\partial q_{i}}=-\left(2 f+c_{i}\right) q_{i}-f q_{-i}+e-b_{i}+\sum_{j \neq i}^{n} \lambda_{j}(-f)=0
$$

In the steady state, $\dot{\lambda}_{j}=0$, then with equation (15), yield:

$$
\begin{aligned}
& \lambda_{j}\left(r+2 f+c_{j}+\frac{\partial u_{j}}{\partial q_{j}}\right)+f \sum_{k \neq j, j \neq i}^{n} \lambda_{k}=-f q_{i} \\
& j=1, \cdots, n, j \neq i
\end{aligned}
$$

For there are $(n-1)$ variables and $(n-1)$ equations, it is possible to solve this equation system. Rewrite equation (17) in the form of matrix:

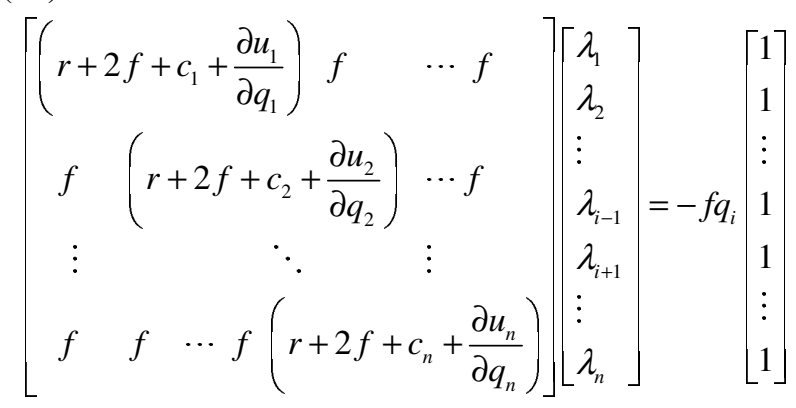

With Guass-Jordan-Elimination, it is easy to solve above equation (18), which yield:

$$
\lambda_{j}=\frac{-f q_{i}}{\left(r+f+c_{j}+\frac{\partial u_{j}}{\partial q_{j}}\right)\left(1+\sum_{k \neq i}^{n} \frac{f}{r+f+c_{k}+\frac{\partial u_{k}}{\partial q_{k}}}\right)}
$$$$
j=1, \cdots, n, j \neq i
$$

From equation (13) and (14), we can obtain:

$$
\frac{\partial H_{i}}{\partial q_{i}}=-\left(2 f+c_{i}\right) q_{i}-f \sum_{j \neq i}^{n} q_{j}+e-b_{i}+\sum_{j \neq i}^{n} \lambda_{j}(-f)=0
$$

In the steady state, $\dot{q}_{i}=0$, i.e.:

$$
e-b_{i}-\left(2 f+c_{i}\right) q_{i}-f \sum_{j \neq i, j=1}^{n} q_{j}-u_{i}=0
$$

From equation (20) and (21), we have:

$$
\begin{aligned}
& u_{i}=\sum_{j \neq i}^{n} \lambda_{j} f \\
& =-f^{2} q_{i} \sum_{j \neq i}^{n} \frac{1}{\left(r+f+c_{j}+\frac{\partial u_{j}}{\partial q_{j}}\right)\left(1+\sum_{k \neq i}^{n} \frac{f}{\left.r+f+c_{k}+\frac{\partial u_{k}}{\partial q_{k}}\right)}\right.}
\end{aligned}
$$

Without loss of generality, we can assume that the discount rate $r$ is very close to zero, and we know that $u_{i}=f C V_{i} q_{i}$, hence obtain:

$$
C V_{i}=-f \sum_{j \neq i}^{n} \frac{1}{\left(f+c_{j}+f C V_{j}\right)\left(1+\sum_{k \neq i}^{n} \frac{f}{f+c_{k}+f C V_{k}}\right)}
$$

$i=1, \cdots, n$

Actually, equation (23) implies lots of interesting information. In what follows, some discussions are given.

Case 1: duopoly case (two suppliers)

Given $n=2$, with (23), yield:

$$
\left\{\begin{array}{l}
C V_{1}=-\frac{f}{2 f+c_{2}+f C V_{2}} \\
C V_{2}=-\frac{f}{2 f+c_{1}+f C V_{1}}
\end{array}\right.
$$

Compared above equation (24) with the equation (10) in reference [9], it can be known that equation (10) in reference [9] can only be correct for duopoly case, and will not hold for a general oligopoly market.

\section{Case 2: perfect competition (infinite supplier)}

Given $n=\infty$, from equation (23), we can have:

$$
\begin{aligned}
& \lim _{n \rightarrow \infty} C V_{i}=\lim _{n \rightarrow \infty} \frac{1}{\left(1+\sum_{k \neq i}^{n} \frac{f}{f+c_{k}+f C V_{k}}\right)} \sum_{j \neq i}^{n} \frac{-f}{\left(f+c_{j}+f C V_{j}\right)} \\
& =\lim _{n \rightarrow \infty} \frac{1}{\sum_{k \neq i}^{n} \frac{f}{f+c_{k}+f C V_{k}}} \sum_{j \neq i}^{n} \frac{-f}{\left(f+c_{j}+f C V_{j}\right)}=-1, \quad i=1, \cdots, n
\end{aligned}
$$

Which means that if there are infinite suppliers in the market, the competition is perfect. This conclusion also matches the economic definition of perfect market.

\section{Case 3: general oligopolistic market}

Any other markets are less competitive than the prefect market, thus for rationality, we should have $C V_{i}>-1(i=1, \cdots, n)$. Furthermore, in electricity markets, the slope of inverse demand function $f$ and production cost function coefficient $c_{i}$ are all positive, thus we have $f+c_{i}+f C V_{i}>0(i=1, \cdots, n)$. Also from equation (23), it is easy to see that the conjecture variation will always be negative, which means that market outcome is more competitive than Cournot $\left(C V_{i}=0\right)$. Finally, for a general oligopoly market, we have:

$$
-1<C V_{i}<0, i=1, \cdots, n
$$

\section{EXISTENCE \& UNIQUENESS OF CONJECTURE VARIATION EQUILIBRIUM}

The next key issue is to show that there is only one equilibrium for the coupled nonlinear equation system given by (23).

For convenience, define a new variable $x_{i}=f+c_{i}+f C V_{i}$ and $y=\sum_{i=1}^{n} \frac{1}{x_{i}}$. We can see that there is one-to-one mapping between the conjecture variation 
$C V_{i}$ and the new variable $x_{i}$. If it can be proved that there is only one equilibrium of $x_{i}$, then it is also equivalent that there is only one conjecture variation equilibrium. And indeed in what follows, it is proved that there is only equilibrium of $x_{i}$.

From equation (23), we obtain:

$$
x_{i}=f+c_{i}+f C V_{i}=f+c_{i}-f^{2} \frac{y-\frac{1}{x_{i}}}{1+f y-\frac{f}{x_{i}}}
$$

Rearrange equation (27), we can get:

$$
(1+f y) x_{i}^{2}-\left(2 f+(1+f y) c_{i}\right) x_{i}+f c_{i}=0
$$

The solutions are:

$$
\begin{aligned}
& x_{i}=\frac{2 f+(1+f y) c_{i} \pm \sqrt{\left[2 f+(1+f y) c_{i}\right]^{2}-4 f(1+f y) c_{i}}}{2(1+f y)} \\
& =\frac{2 f+(1+f y) c_{i} \pm \sqrt{\left[(1+f y) c_{i}\right]^{2}+4 f^{2}}}{2(1+f y)}
\end{aligned}
$$

With the inequality (26), we have $x_{i} \geq c_{i}$, and thus get the right solution as:

$$
x_{i}=\frac{2 f+(1+f y) c_{i}+\sqrt{\left[(1+f y) c_{i}\right]^{2}+4 f^{2}}}{2(1+f y)}
$$

Define a function:

$$
G(y)=\sum_{i=1}^{n} \frac{1}{x_{i}}=\sum_{i=1}^{n} \frac{2(1+f y)}{2 f+(1+f y) c_{i}+\sqrt{\left[(1+f y) c_{i}\right]^{2}+4 f^{2}}}
$$

It is easy to see that $G(y)$ is continuous and differentiable. To show that there exists at least one equilibrium, it is equivalent to show that there exists at least one fixed point $y^{*}$, such that $G\left(y^{*}\right)=y^{*}$.

The parameter $f$ is always positive and generally the parameter $c_{i}$ is positive too, thus we can have:

$$
\begin{aligned}
& G(y)=\sum_{i=1}^{n} \frac{2(1+f y)}{2 f+(1+f y) c_{i}+\sqrt{\left[(1+f y) c_{i}\right]^{2}+4 f^{2}}} \\
& \leq \sum_{i=1}^{n} \frac{2(1+f y)}{2(1+f y) c_{i}}=\sum_{i=1}^{n} \frac{1}{c_{i}}
\end{aligned}
$$

and

$$
\begin{aligned}
& G(y) \geq \sum_{i=1}^{n} \frac{2(1+f y)}{2(1+f y) c_{i}+4 f} \\
& =\sum_{i=1}^{n} \frac{1}{c_{i}+\frac{2 f}{1+f y}} \geq \sum_{i=1}^{n} \frac{1}{c_{i}+2 f}
\end{aligned}
$$

Denoting $S=\left[\sum_{i=1}^{n} \frac{1}{c_{i}+2 f}, \sum_{i=1}^{n} \frac{1}{c_{i}}\right]$, we know that $S$ is closed, convex and compact set, therefore for $y \in S$, we also have $G(y) \in S$. That is to say, the function $G(y)$ is a continuous mapping from $S$ to $S$, therefore, according to Brower's fixed-point theory, there exists at least a point $y^{*} \in S$, such that:

$$
y^{*}=G\left(y^{*}\right)
$$

I.e., there exists at least one equilibrium.

Thus in equilibrium:

$$
c_{i} \leq x_{i}^{*} \leq c_{i}+f
$$

I.e.:

$$
\sum_{i=1}^{n} \frac{1}{c_{i}+f} \leq y^{*} \leq \sum_{i=1}^{n} \frac{1}{c_{i}}
$$

Without changing the existence of equilibrium, we can compress the compact set $S$ to a new compact set $S_{1}=\left[\sum_{i=1}^{n} \frac{1}{c_{i}+f}, \sum_{i=1}^{n} \frac{1}{c_{i}}\right]$. Differentiate $G(y)$, it yields:

$G^{\prime}(y)$

$$
=\sum_{i=1}^{n} \frac{\left[4 f^{2} \sqrt{\left[(1+f y) c_{i}\right]^{2}+4 f^{2}}+8 f^{3}\right]}{\left[(1+f y) c_{i}+2 f+\sqrt{\left[(1+f y) c_{i}\right]^{2}+4 f^{2}}\right]^{2} \sqrt{\left[(1+f y) c_{i}\right]^{2}+4 f^{2}}}
$$

Very clearly, it is know that:

$$
G^{\prime}(y)>0
$$

And it is very easy to check that:

$$
G^{\prime \prime}(y)<0
$$

Thus we know that $G(y)$ is a concave function of $y$.

Lemma 1: (1) when $n=1$, we have one unique equilibrium $x_{1}=c_{1}+f$; (2) when $n \geq 2$, given $y=y_{\min }=\sum_{i=1}^{n} \frac{1}{c_{i}+f}$, for the solution given by (24), we can have $x_{i}<c_{i}+f, \mathrm{i}=1, \cdots, \mathrm{n}$, and $G\left(y_{\text {min }}\right)>y_{\text {min }}$ (i.e., in figure $1, A$ is about $A_{1}$ ).

The proof of lemma 1 can be found in Appendix at the end of this paper.

From the concave property of function $G(y)$, knowing that in figure $1 \mathrm{~A}$ is above $\mathrm{A}_{1}$ and $\mathrm{B}$ should be lower or equal to $\mathrm{B}_{1}\left(\because G(y) \leq \sum_{i=1}^{n} \frac{1}{c_{i}}\right)$, we can conclude that the there is no more than one fixed point $y^{*} \in S_{1}$ such

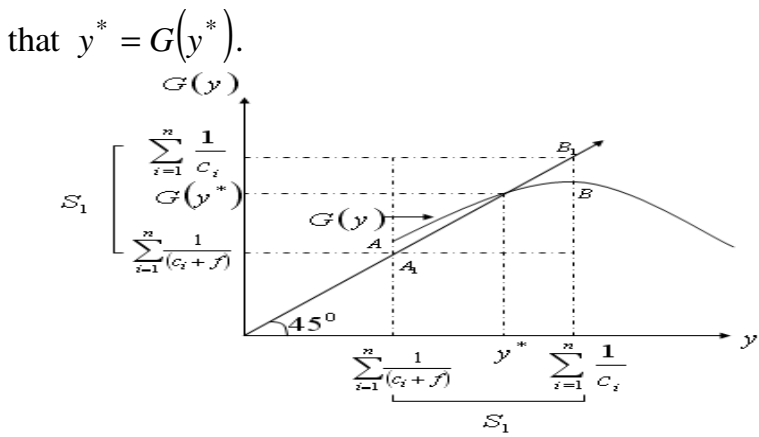

Figure 1 Existence and uniqueness of equilibrium 
Although the issue of existence and uniqueness of equilibrium is well addressed, it is still difficult to calculate the equilibrium directly. For convenience of equilibrium computation, the following dynamic adjustment process can be used in the iteration:

$$
C V_{i}[t]=C V_{i}[t-1]+\partial_{i}\left[-f \sum_{j \neq i}^{n} \frac{1}{\left(f+c_{j}+f C V_{j}\right)\left(1+\sum_{k \neq i}^{n} \frac{f}{f+c_{k}+f C V_{k}}\right)}-C V_{i}[t-1]\right]
$$

\section{NUMERICAL RESULTS}

The IEEE 6-generator 30-bus system is used to verify the above analysis. The inverse demand function in the electricity market is given as:

$$
p=50-0.02 D=50-0.02 \sum_{i=1}^{6} q_{i}
$$

The cost function parameters of suppliers (generators) are listed in Table 1

\section{TABLE I}

Cost function coefficients

\begin{tabular}{|c|c|c|c|}
\hline Supplier & $a_{i}$ & $b_{i}$ & $c_{i}$ \\
\hline 1 & 0 & 2 & 0.02 \\
\hline 2 & 0 & 1.75 & 0.0175 \\
\hline 3 & 0 & 3 & 0.025 \\
\hline 4 & 0 & 3 & 0.025 \\
\hline 5 & 0 & 1 & 0.0625 \\
\hline 6 & 0 & 3.25 & 0.00834 \\
\hline
\end{tabular}

The discount rate $r$ is set to be zero. With iteration, the dynamic process (40) is used to calculate the unique equilibrium. Table 2 shows the equilibrium results:

Table 2 Computation results: generation, profits, $\mathrm{CV}$

\begin{tabular}{cccc}
\hline & $\begin{array}{c}\text { CV } \\
\text { value }\end{array}$ & $\begin{array}{c}\text { Gen- } \\
(\mathrm{MW} / \mathrm{H})\end{array}$ & $\begin{array}{c}\text { Profits } \\
(\$ / \mathrm{H})\end{array}$ \\
\hline Supplier 1 & -0.80725 & 353.4 & 1727.4 \\
\hline Supplier 2 & -0.80365 & 405.12 & 2076.6 \\
\hline Supplier 3 & -0.81241 & 258.44 & 1082.9 \\
\hline Supplier 4 & -0.81241 & 258.44 & 1082.9 \\
\hline Supplier 5 & -0.82528 & 142.9 & 707.48 \\
\hline Supplier 6 & -0.77609 & 560.18 & 2709.8 \\
\hline
\end{tabular}

The total generation is 1978.5 (MW), and the MCP is 10.431(\$MWH).

The following Table 3 shows the computation results when all suppliers are price-taker (i.e., $\mathrm{CV}=-1$ )

Table 3 Computation results with all price-taker suppliers: generation, profits, $\mathrm{CV}$

\begin{tabular}{lccc}
\hline & $\begin{array}{c}\text { CV } \\
\text { value }\end{array}$ & $\begin{array}{c}\text { Gen- } \\
(\mathrm{MW} / \mathrm{H})\end{array}$ & $\begin{array}{c}\text { Profits } \\
(\$ / \mathrm{H})\end{array}$ \\
\hline Supplier 1 & -1 & 348.43 & 1214 \\
\hline Supplier 2 & -1 & 412.49 & 1488.8 \\
\hline Supplier 3 & -1 & 238.74 & 712.47 \\
\hline
\end{tabular}

\begin{tabular}{lccc}
\hline Supplier 4 & -1 & 238.74 & 712.47 \\
\hline Supplier 5 & -1 & 127.5 & 507.98 \\
\hline Supplier 6 & -1 & 685.68 & 1960.5 \\
\hline
\end{tabular}

The total generation is $2051.6(\mathrm{MW})$, and the MCP is 8.9685(\$/MWH).

Table 4 shows the computation results when all suppliers are Cournot-taker (i.e., $\mathrm{CV}=0$ )

Table 4 Computation results with all Cournot-taker suppliers: generation, profits, $\mathrm{CV}$

\begin{tabular}{cccc}
\hline & $\begin{array}{c}\text { CV } \\
\text { value }\end{array}$ & $\begin{array}{c}\text { Gen } \\
(\mathrm{MW} / \mathrm{H})\end{array}$ & $\begin{array}{c}\text { Profits } \\
(\$ / \mathrm{H})\end{array}$ \\
\hline Supplier 1 & 0 & 319.06 & 3054 \\
\hline Supplier 2 & 0 & 347 & 3461.7 \\
\hline Supplier 3 & 0 & 261.39 & 2220.5 \\
\hline Supplier 4 & 0 & 261.39 & 2220.5 \\
\hline Supplier 5 & 0 & 166.82 & 1426.2 \\
\hline Supplier 6 & 0 & 406.23 & 3988.5 \\
\hline
\end{tabular}

The total generation is 1761.9 (MW), and the MCP is 14.76 (\$/MWH).

Compared these results, it is easy to see that each supplier holds some market power, and the market equilibrium results are less competitive than the perfect competition case (less generation and larger MCP). But compared with the dispatched results from the Cournot, the market equilibrium results are more competitive (more generation and smaller MCP). Therefore, we can see that the simulation results support the above analysis very well.

\section{CONCLUSIONS}

The traditional conjecture variation approach for the analysis of strategic behavior in the oligopolistic markets has been criticized widely for some common drawbacks: (1) inconsistent conjecture variation; (2) abundant of equilibrium. Given this background, this paper studies the dynamic oligopolistic competition to explore the unique property of consistent conjecture variation equilibrium in electricity markets. After taking the advantage of some good characteristics of electricity markets, such as the increasing cost function and downslope demand function, it is shown that the consistent conjecture variation should satisfy a coupled nonlinear equation system and with the fixed-point theory, it is proved that there is only one unique conjecture variation equilibrium, and in equilibrium, it results a more competitive behavior than the Cournot. The simulation results support the analysis very well.

\section{APPENDIX}

Lemma 1: (1) when $n=1$, we have one unique equilibrium $x_{1}=c_{1}+f$; (2) when $n \geq 2$, given $y=y_{\min }=\sum_{i=1}^{n} \frac{1}{c_{i}+f}$, for the solution given by (13), we 
can have $x_{i}<c_{i}+f, \mathrm{i}=1, \cdots, \mathrm{n}$, and $G\left(y_{\text {min }}\right)>y_{\text {min }}$.

Proof: (1) when $n=1$, it is the extreme case of monopoly competition, thus clearly we have one unique solution $x_{1}=c_{1}+f$.

(2) when $n \geq 2$, the solution given by (13) is :

$$
x_{i}=\frac{\left(f y c_{i}+2 f+c_{i}\right)+\sqrt{\left(f y c_{i}+c_{i}\right)^{2}+4 f^{2}}}{2(f y+1)}, i=1, \cdots, n(\mathrm{~A}-1)
$$

In order to show:

$$
x_{i}<c_{i}+f, i=1, \cdots, n
$$

the equivalent condition is:

$$
\left(f y c_{i}+2 f+c_{i}\right)+\sqrt{\left(f y c_{i}+c_{i}\right)^{2}+4 f^{2}}<2(f y+1)\left(f+c_{i}\right)(\mathrm{A}-3)
$$

Rearrange it, yield:

$$
\sqrt{\left(f y c_{i}+c_{i}\right)^{2}+4 f^{2}}<\left(f y c_{i}+c_{i}\right)+2 f^{2} y
$$

Then we have:

$$
1<y\left(f y\left(f+c_{i}\right)+c_{i}\right)
$$

Given $y=y_{\min }=\sum_{i=1}^{n} \frac{1}{c_{i}+f}$, it is clear that:

$$
y_{\min }\left(f+c_{i}\right)=\left(f+c_{i}\right) \sum_{i=1}^{n} \frac{1}{c_{i}+f}>\frac{\left(c_{i}+f\right)}{c_{i}+f}=1
$$

Thus:

$$
y_{\text {min }}\left(f y_{\text {min }}\left(f+c_{i}\right)+c_{i}\right)>y_{\text {min }}\left(f+c_{i}\right)>1
$$

i.e., inequality (A-5) will hold and equivalently inequality (A-2) will hold, therefore, given $y=y_{\min }=\sum_{i=1}^{n} \frac{1}{c_{i}+f}$, we can have:

$$
G\left(y_{\min }\right)=\sum_{i=1}^{n} \frac{1}{x_{i}}>\sum_{i=1}^{n} \frac{1}{c_{i}+f}=y_{\min }
$$

\section{REFERENCE}

[1] A.K.David, F.S.Wen, "Strategic bidding in competitive electricity markets: A literature survey", Proceedings of IEEE PES 2000 Summer Power Meeting; pp.2168-2173.

[2] Jong-Bae Park, Kim B.H., Jin-Ho Kim, Man-Ho Jung, Jong-Keun Park, "A continuous strategy game for power transactions analysis in competitive electricity markets", IEEE Trans. Power Syst, Vol.16, No2.pp.847-855,2001.

[3] DelaTorre S., Contreras J., Conejo A.J. "Finding Multiperiod Nash Equilibria in Pool-Based Electricity Markets", IEEE Trans. Power Syst, Vol.19, No.1, pp. 643-651.

[4] James D.Weber, Thomas J.Overbye, "An individual welfare maximization algorithm for electricity markets", IEEE Trans. Power Syst, Vol.17, No.3, pp. 590-596

[5] H. Song, C.-C. Liu, and J. Lawarrée, "Nash equilibrium bidding strategies in a bilateral electricity market", IEEE Trans. Power Syst, Vol.17, pp.73-79, 2002.

[6] Garcia-Alcade A, Ventosa M, Rivier M, Romos A, Relano G., "Fitting electricity market models: a conjectural variations approach", Power System Computation Conference 2002.

[7] Day CJ, Hobbs BF, Pang J., " Oligopolistic competition in power networks: a conjectured supply function approach", IEEE Trans. Power Syst , Vol.17, No3, pp. 597-607,2002.

[8] Song Y, Ni Y, Wen F, Hou Z, Wu FF, “Conjectural variation based bidding strategy in spot markets: fundamentals and comparison with classical game theoretical bidding strategies", Electric Power System Research, Vol.67, No.1 , pp. 45-51, 2003.
[9] Song Y, Ni Y, Wen F, Hou Z, Wu FF, "Conjectural variation based learning model of strategic bidding in spot markets", Electricity Power and Energy Systems, Vol.26, pp.797-804,2004.

[10] Morton I. Kamien, Nancy L. Schwartz, "Conjectural variations", The Canadian Journal of Economics, Vol.16, No.2, pp.191211,1983

[11] Bresnahan T.F, "Duopoly Models with Consistent Conjectures.", American Economic Review, Vol. 71, pp.934-945,1981.

[12] Perry M.K, "Oligopoly and consistent Conjectural variations", Bell journal of Economics, Vol.13, pp.197-205, 1982.

[13] Boyer M., Moreaux M, "Conjectures, Rationality and Duopoly Theory", International Journal of Industrial Organization, Vol.1, pp.1:1-22, 1983

[14] Dockner E.J, "A Dynamic theory of conjectural variations.", Journal of Industrial economics, Vol.4, pp.377-395,1992.

\section{BIOGRAPHIES}

Youfei Liu received the B.S. and M.S. degrees in control theory and control engineering from University of Science and Technology of China (USTC) in 1999 and 2002, respectively. Now he is a PHD candidate in EEE Dept., Hong Kong University. His current research interest is the analysis of dynamics and stability of power market.

Yixin Ni (SM'94) received her B. Eng., M. Eng., and Dr. Eng. degrees all in electrical engineering, from Tsinghua University, China. She was a former Professor and Director of National Power System Lab, Tsinghua University and now is with the University of Hong Kong. Her interests are power system stability and control, FACTS, AI applications in power systems and power markets. 